\title{
Twin registers in Europe: an overview
}

\author{
Dorret I Boomsma \\ Department of Psychology, Vrije Universiteit, Amsterdam, The Netherlands
}

\begin{abstract}
This paper is the first in a series that aims to give an overview of existing twin registers worldwide. A short description of 16 registries in nine European countries is presented. These registries have access to over 350000 twin pairs and are a major resource for genetic-epidemiological research.
\end{abstract}

Keywords: genetic epidemiology, twins, multiples, databases

This paper gives an overview of European twin registers. Taken together, these registers give access to more than 350000 twin pairs of all ages. They are a major resource for genetic-epidemiologal studies of a wide range of phenotypes. Table 1 gives a concise summary of the European twin registers. Appendices 1-16 provide more detailed information on the individual registries and registers.

Table 1 European twin registers

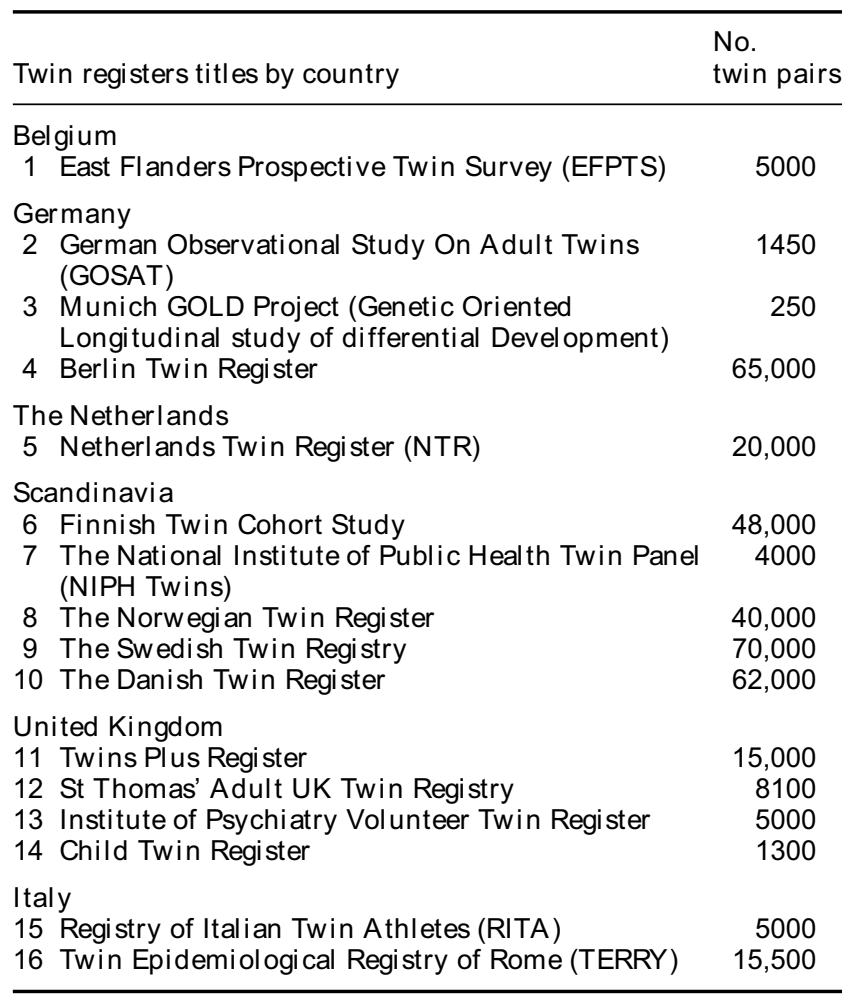

Correspondence: Vrije Universiteit, Dept of Psychology, De Boelelaan 1111, $1081 \mathrm{HV}$ Amsterdam, The Netherlands. Tel: 31 204448787; Fax: 31 204448832; E-mail dorret@sy.vu.nl. Received 26 December 1997; accepted 27 December 1997
The large population-based twin registers in Scandinavia are well known, but large population-based or volunteer twin registers now also exist in Germany, Belgium, the UK, Italy and The Netherlands.

Some registers lack large numbers of participating twin pairs, but have other unique features. The Munich GOLD Project, for example, includes Iongitudinal data over a 60-year period for 90 twin pairs who were first tested in 1937; in Italy a large register of twin athletes has been established; in The Netherlands the NTR has collected longitudinal data of 10000 twin pairs from birth; the EFPTS in Belgium has collected data on placentation on a substantial number of twin pairs in the register and St Thomas' registry relies mainly on clinical investigations such as X-rays and MRI scans.

Evidence of genetic influences on disease, behaviour and other traits can be obtained from the classical twin design in which the resemblance of genetically identical, monozygotic, twins is compared to the resemblance of fraternal, or dizygotic, twins. For some rare diseases a genetic component of disease vulnerability can only be established if sufficient numbers of mono- and dizygotic twins can be studied where at least one member of a pair is affected. For such projects, the value of large twin registers is becoming increasingly clear.

Some rare conditions even need affected twins to be pooled from several registers. For example, a current study of cleft lip is collecting data on twins with cleft lip from eight registers (Mitchell, personal communication).

Most registers have assembled data from questionnaires directed at large numbers of twin pairs. The availability of phenotypic data on large numbers of dizygotic twin pairs offers the possibility of phenotypic screening to ascertain extreme concordant and discordant scoring sibling pairs, who are very informative for linkage analysis of quantitative traits. Compared with ordinary siblings, dizygotic twins have the advantage of being the same age and are less likely to have different fathers, a potential problem in other sibling studies. ${ }^{1}$ 
Findings of significant heritability in one population may not be extrapolated from a second population in which there are differences in exposure to the relevant environmental factors. Replication of twin studies in different countries provides one way to study such interactions between genes and environment. Unfortunately, twin registers from southern European countries are currently under represented.

\section{References}

1 Martin N, Boomsma D, Machin G. A twin-pronged attack on complex traits. Nat Genet 1997; 17: 387-391.

2 Derom C, Vlietinck R, Derom R, Van den Berghe H, Thiery M. Increased monozygotic twinning rate after ovulation induction. Lancet 1987; 1: 1236-1238.

3 Derom C, Vlietinck R, Derom R, Van den Berghe J, Thiery M. Population-based study of sex proportion in monoamniotic twins. N Engl J Med 1988; 319: 119-120.

4 Riemann R, Angleitner A, Strelau J. Genetic and environmental influences on personality: a study of twins reared together using the self- and peer report NEO-FFI scales. Journal of Personality 1997; 65: 449-475.

5 Spinath FM, Riemann R, Hempel S, Schlangen B, Weiss R, Borkenau P, Angleitner A. A day in the life: description of the German Observational Study on Adult Twins (GOSAT) assessing twin similarity in controlled laboratory settings. In: Merviel de I, Deary I, DeFruyt F, Ostendorf F. (eds). Personality Psychology in Europe 7. Tilburg University Press, The Netherlands.

6 Weinert FE (ed). GOLD: Genetic oriented life span study in differential development. Paper 16. Max Planck Institute for Psychological Research: Munich, Germany. 1997.

7 Weinert FE, Geppert U, Doerfert J, Viek P. Aufgaben, Ergebnisse und Probleme der Zwillingsforschung - dargestellt am Beispiel der Gottschaldtschen Längsschnittstudie [Tasks, results and problems of twin research - a description using Gottschaldt's Iongitudinal study as example]. Zeitschrift für Pädagogik. 1994; 40: 265-288.

8 Busjahn A, Knoblauch $\mathrm{H}$, Knoblauch M, Bohlender J, Menz M, Faulhaber HD, Becker A, Schuster H, Luft FC. Angiotensinconverting enzyme and angi otensinogen gene polymorphisms, plasma levels, cardiac dimensions. A twin study. Hypertension 1997; 29(1 Pt 2): 165-170.

9 Busjahn A, Faulhaber HD, Viken RJ, Rose RJ, Luft FC. Genetic influences on blood pressure with the cold-pressor test: a twin study. J Hypertens 1994; 14: 1195-1194.

10 Boomsma DI, Orlebeke F, Baal GCM van. The Dutch Twin Register: growth data on weight and height. Behav Genet 1992; 22: $247-251$

11 Koopmans R, Doornen LJP van, Boomsma DI. Association between alcohol use and smoking in adolescent and young adult twins: a bivariate genetic analysis. Alcoholism: Clinical and Experimental Research 1997: 21: 537-546.

12 Kaprio J, Koskenvuo M, Rose RJ. Population-based twin registries: illustrative applications in genetic epidemiology and behavioural genetics from the Finnish Twin Cohort Study. Acta Genet Med Gemellol 1990; 39: 427-439.

13 Kaprio J. Lessons from twin studies in Finland. Annals of Medicine 1994; 26: 135-139.

14 Harris R, Tambs K, Magnus P. Sex-specific effects for body mass index in the new Norwegian twin panel. Genet Epidemiol 1995; 12: 251-265.
15 Tambs K. Harris R, Magnus P. Sex-specific causal factors and effects of common environment for anxiety and depression in twins. Behav Genet 1995; 25: 33-44.

16 Kringlen E. Norwegian twin registers. Nance WE (ed.) In: Twin Research: Biology and Epidemiology Alan R. Liss: New York; 1978.

17 Ahlbom A, Lichtenstein P, Malmström H, Feychting $M$, Hemminki K, Pedersen NL. Cancer in twins: genetic and nongenetic familial risk factors. J Natl Cancer Inst 1997; 89: 287-293.

18 Cederlof R, Lorich U. The Swedish Twin Registry. Nance WE, Allen G, Parisi P. (eds). In: Twin Research: Biology and epidemiology. Alan R. Liss: New York. 1978; pp 189-195.

19 Kyvik KO, Christensen K, Skytthe A, Harvald B, Holm NV. The Danish Twin Register. Dan Med Bull 1996; 43: 467-470.

20 Christensen K, Vaupel J, Holm NV, Yashin AI. Twin mortality after age 6: fetal origins hypothesis versus twin method. Brit Med J 1995; 310: 432-436.

21 Eley TE, Rutter M, Simonoff E, Bishop DVM, Dale PS, Oliver B, Petrill SA, Price T, Saudino KJ, Stevenson J, Taylor E. Plomin R. Genetic influence on slow language development in 2-year-olds. Submitted.

22 Dale PS, Rutter M, Simonoff E, Bishop DVM, Eley T, Oliver B, Petrill SA, Price T, Purcell S, Saudino KJ, Stevenson J, Taylor E. Plomin R. Genetic influence on slow language development in 2-year-olds. Submitted.

23 Spector TD, Ciccutini F, Baker J, Louglin J, Hart DI. Genetic influences on osteoarthritis in women: a twin study. Br Med J 1996; 312: 940-943.

24 MacGregor AJ, Griffiths GD, Baker J, Spector TD. Determinants and pressure pain threshold in adult twins: evidence that shared environmental effects predominate. Pain 1997; 73: 253-257.

25 Macdonald AM, Harner S. Development of Computerised Storage Facilities for Twin Data: A Relational Database System for A Twin Register. Behav Genet 1997; 27: 1-13.

26 Easton DF, Cox GM, Macdonald AM, Ponder BAJ. The study of naevi in British twins: study design and description of the data set. Cytogenetics and Cell Genetics 1992; 59: 165-166.

27 Stevenson J, Fielding J. Ratings of temperament in families of young twins. Brit J Develop Psychol 1985; 3: 143-152.

28 Stevenson J, Batten N, Cherner M. Fears and fearfulness in children and adolescents: a genetic analysis of twin data. $J$ Child Psychol Psychiatry 1992; 33: 977-186.

29 Parisi P, Casini B, Pittaluga M, Di Salvo V, Pigozzi F, Cilia G, Kussouras $V$. Twin registers for sport science: a unique resource for the assessment of genetic and environmental effects. Proceedings of the First Annual Congress of the European College of Sport Science, Nice. 1996.

30 Parisi P, Casini B, Pittaluga M, Prinzi G, Di Salvo V, Pigozzi F, Klissouras V. The registry of Italian twin athl etes (RITA). Pilot study: establishment of a panel of twin swimmers. Proceedings of the Second Annual Congress of the European College of Sport Science, Copenhagen. 1997. 
Appendix 1

Name of register

Country

Birth cohorts

How are twins

ascertained?

Opposite sex twins included?

Number of twin pairs in each age range

Primary interests/ variables measured

Institution

Contact

Address

Tel.

Fax

E-mail
East Flanders Prospective Twin Survey (EFPTS)2, 3

Belgium

July 1964 onwards

At birth, prospectively and population based

the EFPTS records all multiples born in East Flanders

yes

Since the start of the register, around 120 twin pairs per year. Since 1985 there has been an increase in the twin pregnancy rate and in 1996 the EFPTS recorded about 260 pairs. as of September 1997 there were 5000 twin pairs and more than 150 higher order multiple births.

Zygosity, placentation (moment of splitting in MZ), congenital anomalies and prenatal factors, cardio-vascular and physical fitness measurements, cognitive development (IQ), Child Behaviour Check List, Iateral isation

Center for Human Genetics, KULeuven

Prof. R. Vlietinck, Dr C. Derom, Prof. R. Derom

Gasthui sberg O\&N, Herestraat 49 B-3000 Leuven, Bel gium

3216345865

3216345994

robert.vlietinck@med.kuleuven.ac.be catherine.derom@med.kuleuven.ac.be robert.derom@ug.ac.be 
Appendix 2

\begin{tabular}{|c|c|}
\hline Name of register & German Observational Study On Adult Twins (GOSAT) ${ }^{4,5}$ \\
\hline Country & Germany \\
\hline Birth cohorts & $\begin{array}{l}\text { 1915-1977 (adult twins) } \\
1978-1996 \text { (twin children) }\end{array}$ \\
\hline $\begin{array}{l}\text { How are twins } \\
\text { ascertained? }\end{array}$ & via newspapers, magazines, twin clubs and twin meetings \\
\hline $\begin{array}{l}\text { Opposite sex } \\
\text { twins included? }\end{array}$ & yes \\
\hline $\begin{array}{l}\text { Number of twin pairs } \\
\text { in each age range }\end{array}$ & $\begin{array}{l}\text { approximately } 1000 \text { adult twins and } \\
\text { approximately } 450 \text { young twins }\end{array}$ \\
\hline \multirow[t]{3}{*}{$\begin{array}{l}\text { Primary interests/ } \\
\text { variables measured }\end{array}$} & $\begin{array}{l}\text { all adult twins: self-report personality and temperament questionnaires; } \\
2 \text { peer reports per twin on most of these questionnaires. }\end{array}$ \\
\hline & $\begin{array}{l}\text { sub-sample of } 300 \mathrm{MZ} \text { and same sex DZ twin pairs: behaviour } \\
\text { observations in quasi-natural situations (videos), psychometric } \\
\text { intelligence data, mental speed data from elementary cognitive tasks, } \\
\text { objective temperamental measures, unobtrusive behaviour registrations } \\
\text { during an assessment day. }\end{array}$ \\
\hline & $\begin{array}{l}\text { twin children: } \\
\text {-parental reports on temperament and personality scal es }\end{array}$ \\
\hline Institution & University of Bielefeld, Department of Differential Psychology \\
\hline Contact & Alois Angl eitner, Rainer Riemann, Frank M. Spinath \\
\hline Address & PO Box 100131, 33501 Biel efeld, Germany \\
\hline Tel. & 495211064456 \\
\hline Fax & 495211066422 \\
\hline E-mail & fspin@post.uni-bielefeld.de \\
\hline
\end{tabular}


Appendix 3

\begin{tabular}{|c|c|}
\hline Name of register & $\begin{array}{l}\text { Munich GOLD Project (Genetic Oriented Longitudinal } \\
\text { study of differential Development) } 6,7\end{array}$ \\
\hline Country & Germany \\
\hline Birth cohorts & approximately 1912-1932 \\
\hline $\begin{array}{l}\text { How are twins } \\
\text { ascertained? }\end{array}$ & new spapers advertisements \\
\hline $\begin{array}{l}\text { Opposite sex } \\
\text { twins included? }\end{array}$ & no \\
\hline $\begin{array}{l}\text { Number of twin pairs } \\
\text { in each age range }\end{array}$ & about 250 identical and fraternal pairs in old age \\
\hline $\begin{array}{l}\text { Primary interests/ } \\
\text { variables measured }\end{array}$ & $\begin{array}{l}\text { personality, intelligence, social behaviour, motivation, emotion, morality. } \\
\text { For } 35 \text { twin pairs longitudinal data for 50-60 years are available (former } \\
\text { Gottschaldt study, started in 1937) }\end{array}$ \\
\hline Institution & Max Planck Institute for Psychological Research \\
\hline Contact & Prof. Dr Franz E Weinert/Dr Ulrich Geppert \\
\hline Address & P.O. Box 440109, D-80750 Munich, Germany \\
\hline Tel. & 498938602220 \\
\hline Fax & 4989342473 \\
\hline E-mail & geppert@mpipf-muenchen.mpg.de \\
\hline URL/website & www.mpipf-muenchen.mpg.de/BCD/PROJECTS/gold_e.htm \\
\hline
\end{tabular}


Appendix 4

\begin{tabular}{|c|c|}
\hline Name of register & Berlin Twin Register 8,9 \\
\hline Country & (East-) Germany \\
\hline Birth cohorts & 1920-1999 \\
\hline $\begin{array}{l}\text { How are twins } \\
\text { ascertained? }\end{array}$ & based on birth registers \\
\hline $\begin{array}{l}\text { Opposite sex } \\
\text { twins included? }\end{array}$ & yes \\
\hline $\begin{array}{l}\text { Number of twin pairs } \\
\text { in each age range }\end{array}$ & about $60-70,000$ total \\
\hline $\begin{array}{l}\text { Primary interests/ } \\
\text { variables measured }\end{array}$ & cardiovascular phenotypes, personality, coping \\
\hline Institution & Franz-Volhard-Klinik/Max-Delbrueck-Center for Molecular Medicine \\
\hline Contact & Andreas Busjahn \\
\hline Address & $\begin{array}{l}\text { Franz-Volhard-Klinik/MDC } \\
\text { Wiltbergstr. } 50,13125 \text { Berlin, Germany }\end{array}$ \\
\hline Tel. & 493094172277 \\
\hline Fax & 493094172335 \\
\hline E-mail & busjahn@vk-berlin.de \\
\hline URL/w ebsite & http://www.fvk-berlin.de/fvkweb/nephrology/index.html \\
\hline
\end{tabular}


Appendix 5

Name of register

Country

Birth cohorts

How are twins

ascertained?

Opposite sex

twins included?

Number of twin pairs in each age range

Primary interests/ variables measured

Institution

Contact person

Address

Tel.

Fax

E-mail

URL/website
Netherlands Twin Register (NTR) ${ }^{10,11}$

The Netherlands

1986-1998 (young twins)

1940-1985 (adolescent and adult twins)

via commercial organisation (young twins)

population registers (adol escent and adult twins)

yes

between 1000 and 1500 pairs for each 1986-97 cohort

3500 adolescent and adult pairs (participating)

3000 adolescent and adult pairs (registered)

cardiovascular risk, including behavioural factors, development of psychopathology in children and adults, brain function and structure, cognition

NTR, Vrije Universiteit

Dorret Boomsma

De Boel elaan 1111, 1081 HV Amsterdam, The Netherlands

31204448789

31204448832

ntr@psy.vu.nl

http://www/psy.vu.nl/ntr 
Appendix 6

\begin{tabular}{|c|c|}
\hline Name of register & Finnish Twin Cohort Study 12,13 \\
\hline Country & Finland \\
\hline Birth cohorts & $\begin{array}{l}\text { before, 1958, like-sex, with both twins alive in } 1967 \text { (old) } \\
\text { 1938-1957, opposite sex } \\
\text { 1958-1986, all multiple births }\end{array}$ \\
\hline $\begin{array}{l}\text { How are twins } \\
\text { ascertained? }\end{array}$ & population registers \\
\hline $\begin{array}{l}\text { Opposite sex } \\
\text { twins included? }\end{array}$ & yes \\
\hline $\begin{array}{l}\text { Number of twin pairs } \\
\text { in each age range }\end{array}$ & $\begin{array}{l}17000 \text { pairs old cohort } \\
8000 \text { pairs older opposite sex pairs } \\
23000 \text { multiple birth sets } 58-86\end{array}$ \\
\hline $\begin{array}{l}\text { Primary interests/ } \\
\text { variables measured }\end{array}$ & $\begin{array}{l}\text { chronic disease risk (cardiovascular, cancer, asthma and other respiratory } \\
\text { disease, sl eep disorders and other neurol ogical, psychopathology) } \\
\text { health-related behaviours, particularly in adolescence }\end{array}$ \\
\hline Institution & Department of Publish Health \\
\hline Contact & Jakkko Kaprio \\
\hline Address & P.O. Box 41, FIN 00014 University of Helsinki, Finland \\
\hline Tel. & 358919127595 \\
\hline Fax & 358919127600 \\
\hline E-mail & Jaakko.Kaprio@Helsinki.fi \\
\hline URL/website & http://kate.pc.helsinki.fi/twin/twinhome.html \\
\hline
\end{tabular}


Appendix 7

Name of register

Country

Birth cohorts

How are twins

ascertained?

Opposite sex

twins included?

Number of twin pairs in each age range

Primary interests/ variables measured
The National Institute of Public Health Twin Panel (NIPH Twins) ${ }^{14,} 15$

\section{Norway}

1967-1974 (questionnaire in 1992, will be recontacted in 1998) 1974-1979 (will be contacted via questionnaire in 1998)

population records from Medical Birth Registry

yes

approximately 640 pairs born each year for birth cohorts from 1967 to end 1974. Questionnaire data is collected from 5864 twins (2570 complete pairs). Sample will be expanded to include birth cohorts from 1975 to end 1979.

sex differences in health and development, body mass, asthma, psychological health variables, physical health history, exercise, smoking, al cohol use, psychological well-being, pre- and peri-natal influences on adult health.

Institution

The National Institute of Public Health, Oslo, Norway

Contact

Jennifer Harris

Address

Department of Population Health Sciences, Section of Epidemiology, Post Box 4404 Torshov, N-0403 Oslo, Norway

Tel.

4722042305

Fax

4722042351

E-mail 
Appendix 8

Name of register

Country

Birth cohorts

How are twins

ascertained?

Opposite sex twins included?

Number of twin pairs between 800 and 900 pairs for each cohort in each age range

Primary interests/ variables measured

Institution

Contact

Address

Tel.

Fax

E-mail
The Norw egian Twin Register ${ }^{16}$

Norway

1895-1944

population registry (Norwegian Statistical Bureau)

yes

mental illness, especially schizophrenia, manic depressive disorder and senile dementia

Department of Psychiatry, University of Oslo

Professor Einar Kringlen

Box 85 Vinderen, 0319 Oslo, Norway

4722146590

4722142911

einar.kringlen@sykiatri.uio.no 
Appendix 9

Name of register

Country

Birth cohorts

How are twins ascertained?

Opposite sex twins included?

Number of twin pairs in each age range

Primary interests/ variables measured

Institution

Contact

Address

Tel.

Fax

E-mail
The Swedish Twin Registry17, 18

Sweden

1886-1925 (old cohort)

1926-1967 (middle cohort)

1968-1990 (young twins)

old cohort manual extraction from church records

middle cohort from birth records

young twins computerised compilation from The Medical Birth Registry

yes

old cohort 11000 (questionnaires on 3 occasions) middle cohort 37500 (1 questionnaire to all like-sexed) young twins 22000 (birth registration information only) some subsamples have participated in numerous questionnaires and/or in person testing data collection

aging, dementia, cognition, personality, depression, cardiovascular diseases, cancer, asthma and allergies, behavioural disorders, health and heal th-related behaviours, gender differences

Institute of Environmental Medicine, Karolinska Institute

Nancy Pedersen, Paul Lichtenstein

Box 210, S171 77 Stockholm, Sweden

4687287418

468304571

nancy.pedersen@mm.ki.se, paul.lichtenstein@mm.ki.se 


\begin{tabular}{|c|c|c|}
\hline Name of register & \multicolumn{2}{|c|}{ The Danish Twin Register ${ }^{19,} 20$} \\
\hline Country & \multicolumn{2}{|l|}{ Denmark } \\
\hline Birth cohorts & 1870-1992 & $1930-52$ in the process of being ascertained \\
\hline $\begin{array}{l}\text { How are twins } \\
\text { ascertained? }\end{array}$ & $\begin{array}{l}1870-1930 \\
1930-1982 \\
1983-1992\end{array}$ & $\begin{array}{l}\text { parish registers } \\
\text { Danish Civil Registration System } \\
\text { Medical Birth Register }\end{array}$ \\
\hline $\begin{array}{l}\text { Opposite sex } \\
\text { twins included? }\end{array}$ & \multicolumn{2}{|l|}{ yes } \\
\hline $\begin{array}{l}\text { Number of twin pairs } \\
\text { in each age range }\end{array}$ & $\begin{array}{l}1870-1930 \\
1930-1952 \\
1953-1982 \\
1983-1992\end{array}$ & $\begin{array}{l}14000 \text { pairs } \\
15000 \text { pairs (anticipated) } \\
20000 \text { pairs } \\
13000 \text { pairs }\end{array}$ \\
\hline $\begin{array}{l}\text { Primary interests/ } \\
\text { variables measured }\end{array}$ & \multicolumn{2}{|c|}{$\begin{array}{l}\text { mortality, life span, causes of death, aging, cancer, diabetes, the insulin } \\
\text { resistance syndrome and related cardiovascular risk factors, clinical } \\
\text { studies of a variety of diseases }\end{array}$} \\
\hline Institution & \multicolumn{2}{|c|}{ Odense University Medical School } \\
\hline Contact & \multicolumn{2}{|c|}{$\begin{array}{l}\text { Kirsten Ohm Kyvik, Niels V. Holm, Kaare Christensen, } \\
\text { Axel Skytthe }\end{array}$} \\
\hline Address & \multicolumn{2}{|c|}{$\begin{array}{l}\text { The Danish Twin Regi ster, Winsloewparken 15, } \\
5000 \text { Odense C, Denmark }\end{array}$} \\
\hline Tel. & \multicolumn{2}{|c|}{4565573047} \\
\hline Fax & \multicolumn{2}{|c|}{4565906531} \\
\hline E-mail & \multicolumn{2}{|c|}{ k-kyvik@vin-chs.ou.dk } \\
\hline
\end{tabular}


Appendix 11

\begin{tabular}{|c|c|}
\hline Name of register & Twins Plus Regi ster21, 22 \\
\hline Country & England and Wales \\
\hline Birth cohorts & $1994,1995,1996$ \\
\hline $\begin{array}{l}\text { How are twins } \\
\text { ascertained? }\end{array}$ & birth records \\
\hline $\begin{array}{l}\text { Opposite sex } \\
\text { twins included? }\end{array}$ & yes \\
\hline $\begin{array}{l}\text { Number of twin pairs } \\
\text { in each age range }\end{array}$ & approximately $5000 /$ cohort (total around 15000 ) \\
\hline $\begin{array}{l}\text { Primary interests/ } \\
\text { variables measured }\end{array}$ & language and cognitive development, behaviour \\
\hline Institution & Institute of Psychiatry \\
\hline Contact & Bonny Oliver \\
\hline Address & 113 Denmark Hill, London SE5 8AF, UK \\
\hline Tel. & 441717405122 \\
\hline Fax & 441719193866 \\
\hline E-mail & spjwbro@op.bpmf.ac.uk \\
\hline
\end{tabular}




\begin{tabular}{|c|c|}
\hline Name of register & St Thomas' Adult UK Twin Registry23, 24 \\
\hline Country & UK \\
\hline Birth cohorts & $1922-1979$ \\
\hline $\begin{array}{l}\text { How are twins } \\
\text { ascertained? }\end{array}$ & media \\
\hline $\begin{array}{l}\text { Opposite sex } \\
\text { twins included? }\end{array}$ & not currently \\
\hline $\begin{array}{l}\text { Number of twin pairs } \\
\text { in each age range }\end{array}$ & $\begin{array}{l}\text { >70 yrs: } 200 \text { pairs; } 60-70: 1000 ; 50-60: 1800 ; 40-50: 2000 \\
\text { 30-40: } 1600 ; 18-30: 1500\end{array}$ \\
\hline $\begin{array}{l}\text { Primary interests/ } \\
\text { variables measured }\end{array}$ & $\begin{array}{l}\text { common diseases of aging; osteoporosis, arthritis, } \\
\text { cardiovascular disease, asthma, blindness, dementia, backpain, } \\
\text { skin diseases, immune function assessed by detailed clinical } \\
\text { examination and investigation and blood phenotype }\end{array}$ \\
\hline Institution & Twin Research Unit, Guy's and St Thomas' Hospital Trust \\
\hline Contact & Tim Spector \\
\hline Address & Lambeth Palace Rd, London SE1 7EH, UK \\
\hline Tel. & $441719289292 \times 2084$ \\
\hline Fax & 441719228234 \\
\hline E-mail & c.manzi@imds.ac.uk \\
\hline URL/website & http://www.umds.ac.uk/twin-unit \\
\hline
\end{tabular}


Appendix 13

\begin{tabular}{|c|c|}
\hline Name of register & Institute of Psychiatry Volunteer Twin Register25, 26 \\
\hline Country & UK \\
\hline Birth cohorts & no \\
\hline $\begin{array}{l}\text { How are twins } \\
\text { ascertained? }\end{array}$ & $\begin{array}{l}\text { via media recruitment (without reference to specific } \\
\text { studies/diseases) }\end{array}$ \\
\hline $\begin{array}{l}\text { Opposite sex } \\
\text { twins included? }\end{array}$ & yes \\
\hline $\begin{array}{l}\text { Number of twin pairs } \\
\text { in each age range }\end{array}$ & 5000 adult twin pairs age range $16-90+$, mean age 34 years \\
\hline $\begin{array}{l}\text { Primary interests/ } \\
\text { variables measured }\end{array}$ & $\begin{array}{l}\text { psychopathology, personality and behavioural aspects of } \\
\text { illness, cognitive function and aging, risk factors for physical } \\
\text { diseases }\end{array}$ \\
\hline Institution & Institute of Psychiatry \\
\hline Contact & Alison Macdonald \\
\hline Address & $\begin{array}{l}\text { Department of Psychological Medicine } \\
\text { De Crespigny Park, London SE5 8AF, UK }\end{array}$ \\
\hline Tel. & 441719193416 \\
\hline Fax & 441717019044 \\
\hline E-mail & spjuamm@op.bpmf.ac.uk \\
\hline
\end{tabular}


Appendix 14

\begin{tabular}{|c|c|}
\hline Name of register & Child Twin Register27, 28 \\
\hline Country & UK \\
\hline Birth cohorts & from 1975 onwards \\
\hline $\begin{array}{l}\text { How are twins } \\
\text { ascertained? }\end{array}$ & volunteers \\
\hline $\begin{array}{l}\text { Opposite sex } \\
\text { twins included? }\end{array}$ & yes \\
\hline $\begin{array}{l}\text { Number of twin pairs } \\
\text { in each age range }\end{array}$ & $\begin{array}{l}\text { born before 1975: } 76 \\
\text { born in 1975-1979: } 286 \\
\text { 1980-1984: } 309 \\
\text { 1985-1989: } 433 \\
\text { after } 1989: 195\end{array}$ \\
\hline $\begin{array}{l}\text { Primary interests/ } \\
\text { variables measured }\end{array}$ & $\begin{array}{l}\text { personality, behaviour problems, psychopathology, social and emotional } \\
\text { development }\end{array}$ \\
\hline Institution & $\begin{array}{l}\text { Centre for Research into Psychological Development, Dept of Psychology, } \\
\text { University of Southampton }\end{array}$ \\
\hline Contact & Prof. Jim Stevenson \\
\hline Address & $\begin{array}{l}\text { Dept of Psychology, University of Southampton, Highfield, } \\
\text { Southampton SO17 1BJ, UK }\end{array}$ \\
\hline Tel. & 441703592583 \\
\hline Fax & 441703594719 \\
\hline E-mail & jsteven@psy.soton.ac.uk \\
\hline URL/website & http://www.soton.ac.uk/ psyweb/staffpages/jsteven/jsteven.html \\
\hline
\end{tabular}


Appendix 15

Name of register

Country

How are twins ascertained?

Opposite sex twins included?

Number of twin pairs in each age range

Primary interests/ variables measured

Institution

Contact

Address

Tel.

Fax

E-mail
Registry of Italian Twin Athletes (RITA) 29, 30

Italy

from registries of the national sports federation

yes

$M M=2600$

$\mathrm{FF}=1500$

$\mathrm{MF}=900$

influence of genetic and environmental factors on high level sport performance

Human Biology and Twin Research Center, ISEF, and Dept of Public Health, University of Tor Vergata, Rome

Prof. Paolo Parisi

Piazza Lauro De Bosis 15, 00194 Rome, Italy

39632650767

39636095562

parisi@itovrm.it 
Appendix 16

\begin{tabular}{|c|c|}
\hline Name of register & Twin Epidemiological Registry of Rome (TERRY) \\
\hline Country & Italy \\
\hline Birth cohorts & $1901-1994$ \\
\hline $\begin{array}{l}\text { How are twins } \\
\text { ascertained? }\end{array}$ & from official records of the resident population \\
\hline $\begin{array}{l}\text { Opposite sex } \\
\text { twins included? }\end{array}$ & yes \\
\hline $\begin{array}{l}\text { Number of twin pairs } \\
\text { in each age range }\end{array}$ & $\begin{array}{l}2100 \text { pairs born } 1901-1944 \\
7300 \text { pairs born } 1945-1969 \\
6100 \text { pairs born } 1970-1994\end{array}$ \\
\hline $\begin{array}{l}\text { Primary interests/ } \\
\text { variables measured }\end{array}$ & $\begin{array}{l}\text { life style (physical activity, nutrition, habits, etc.) and heal th, with special } \\
\text { regard to major risk factors and the aging process }\end{array}$ \\
\hline Institution & $\begin{array}{l}\text { Human Biology and Twin Research Center, ISEF, and Dept } \\
\text { of Public Health, University of Tor Vergata, Rome }\end{array}$ \\
\hline Contact & Prof. Paolo Parisi \\
\hline Address & Piazza Lauro De Bosis 15, 00194 Rome, Italy \\
\hline Tel. & 39632650767 \\
\hline Fax & 39636095562 \\
\hline E-mail & parisi@itovrm.it \\
\hline
\end{tabular}

